\title{
Elementos para Comprender la Concentración de Estudiantes Extranjeros en Escuelas Chilenas
}

\section{Elements to Understand the Clustering of Foreign Students in Chilean Schools}

\author{
Claudia Córdoba $*_{1}$ \\ Carolina Altamirano ${ }^{2}$ \\ Karina Rojas ${ }^{3}$ \\ ${ }^{1}$ Universidad de Santiago de Chile, Chile \\ ${ }^{2}$ Municipalidad de Estación Central, Chile \\ ${ }^{3}$ Universidad Rey Juan Carlos, España
}

\begin{abstract}
La migración hacia Chile se ha incrementado notablemente en los últimos años lo que impacta en la cantidad de estudiantes extranjeros que atiende el sistema escolar. Éstos, a diferencia de sus pares chilenos, asisten en mayor medida a escuelas municipales (públicas), sin embargo, se constata que la proporción de estudiantes extranjeros que son atendidos por este tipo de escuela es muy diversa aun cuando se ubiquen en un mismo territorio. Además, sólo algunas escuelas llegan a concentrar a este alumnado lo que habitualmente es explicado en función de la gratuidad y escasas barreras de ingreso que caracterizan a las escuelas municipales, así como por el patrón residencial de la población migrante. La concentración de estudiantes extranjeros resulta relevante para el chileno por representar una nueva forma de segregación escolar en un sistema especialmente segmentado en términos socioeconómicos y académicos. El artículo presenta los resultados de un estudio etnográfico desarrollado con dos escuelas municipales vecinas y que exhiben distinta proporción de alumnado extranjero. Los resultados sugieren que las familias jugarían un rol protagónico en la concentración porque tienden a auto segregarse con el objeto de proteger a sus hijos de situaciones de discriminación que se asocien a su condición de migrante.
\end{abstract}

Descriptores: Escuela pública; Segregación escolar; Elección de la escuela, Migración internacional; Chile.

\begin{abstract}
Migration to Chile has incremented significantly during the last few years, which has an impact on the quantity of foreign students registered in the school system. These kind of students, unlike the Chilean ones, mainly attend public schools. However, it is established that the percentage of foreign students that go to this type of school also varies even when they are in the same area. Moreover, only some schools concentrate foreign students. This is usually explained by the fact that public schools are free of charge and there are few barriers to control the admission and, also, because of the migrant population residential pattern. The concentration of foreign students is relevant to Chilean school system because it represents a new form of segregation in a system already fragmented in a socioeconomic and academic level. This article presents the results of an ethnographic study developed in two nearby public schools. Both show a variation of foreign student percentages. The results show that families play a vital role because they tend to self-segregate themselves to protect their children from discriminatory situations related to their migrant condition.
\end{abstract}

Keywords: Public schools; School segregation; Choice of school; International migration; Chile.

*Contacto: claudia.cordoba.c@usach.cl

Recibido: 10/07/2019

$1^{\text {a }}$ Evaluación: 11/08/2019

ISSN: 0718-7378

Aceptado: 11/11/2019

www.rinace.net/rlei/ 


\section{Introducción}

Desde la década de los '90 Chile comienza a posicionarse como un país atractivo para migrantes provenientes de otros países de América Latina. Las razones que explican el fenómeno son variadas: intensificación de regulaciones impuestas por destinos tradicionales (Estados Unidos y Europa), profundización de condiciones de pobreza, estabilidad política junto con sus indicadores económicos que muestran una tasa de crecimiento positiva y bajos niveles de desempleo en Chile (Galaz, Poblete y Frías, 2017; Rojas y Silva, 2016).

Las estimaciones más recientes, que consideran tanto cifras censales como registros administrativos, indican que la cifra actual de extranjeros residentes en Chile asciende a 1.251.225 (INE-DEM, 2019). De acuerdo a la Encuesta CASEN 2017, el 93,7\% de ellos proviene de países latinoamericanos y del Caribe: Venezuela, Perú, Colombia, Haití, Bolivia, Argentina y Ecuador (Ministerio de Desarrollo Social s/f, CASEN, 2017). Se destaca de forma especial el caso haitiano, cuyos residentes se incrementan en un $731 \%$ entre 2002 y 2016 , período en que ingresaron al país 41.000 personas de esta nacionalidad (Sánchez et al., 2018)

En relación con la distribución de esta población en el territorio un 71,8\% se concentra en la región Metropolitana, mientras la macrozona norte (que agrupa las regiones de Arica y Parinacota, Tarapacá, Antofagasta y Atacama) aglutina un 12,6\% del (CASEN, 2017; Ministerio de Desarrollo Social, s/f). Junto a ello se aprecia que ciertos colectivos se encuentran más representados en unas regiones que en otras. En la macrozona norte predomina población boliviana, peruana y colombiana, mientras en la Región Metropolitana destaca la presencia venezolana, peruana y colombiana (Ministerio de Desarrollo Social, s/f, CASEN 2017). Asimismo, se constata que la población extranjera habita en algunas comunas y más específicamente en determinados barrios, lo que se explica por diversas razones: precio de la vivienda, conectividad, presencia de actividades productivas y/o existencia de una red de intercambio de información (Margarit y Galaz, 2018).

Un 60\% de los inmigrantes residentes en Chile se ubica en el tramo de edad 20 a 39 años representando los niños, niñas y jóvenes entre o y 19 años una menor proporción dentro del total (14,7\%) (INE-DEM, 2019). Los datos de la Encuesta CASEN 2017 indican que un $12,7 \%$ de la población extranjera residente tiene entre 0 y 14 años: $14,3 \%$ son varones y 11,2 son niñas. Aun cuando esta población aparezca como minoritaria en el conjunto total de extranjeros residentes en Chile, es indudable que su llegada ha impactado al sistema escolar chileno debido a su incorporación sostenida y rápida. Por ejemplo, el sistema escolarizó en 2017 a un total de 76.813 estudiantes extranjeros incrementándose más del doble para 2019 en que la cifra fue de 160.461 estudiantes extranjeros (MINEDUC - CEM, 2019).

Este alumnado asiste preferentemente a establecimientos municipales: $59,1 \%$, seguido de un $36,5 \%$ que lo hace a escuelas privadas subvencionadas. Sólo un 4,5\% se escolariza en escuelas privadas no subvencionados (MINEDUC-CEM, 2019). En cuanto a su distribución por nivel de enseñanza, la mayor parte de ellos se encontraba cursando enseñanza básica en 2017: 62.2\%, seguido de enseñanza media con un 24,3\% y un 13,5\% en enseñanza prebásica (Córdoba y Miranda, 2018). 
Siguiendo la tendencia descrita para la población migrante adulta, los estudiantes extranjeros se concentran en mayor medida en determinadas regiones y, dentro de ellas, en ciertas comunas (MINEDUC, 2017). Sin embargo, incluso dentro de ellas se observan diferencias ostensibles en la proporción que puede ser atendida por escuelas municipales (Castillo, Santa Cruz y Vega, 2018). En efecto, la distribución de este alumnado no es igual entre este tipo de escuelas en las comunas que los concentran en mayor medida, de manera que mientras algunas escolarizan a proporciones muy pequeñas, otras los acogen en tasas iguales o superiores al 30\% (Córdoba y Miranda, 2018).

Se observa entonces una distribución desigual de este alumnado entre las escuelas municipales disponibles lo que estaría dando cuenta de cierto grado de segregación en esta población. Este fenómeno resulta de especial interés para el sistema escolar chileno que, en perspectiva internacional, destaca por su alto grado de segregación socioeconómica (OCDE, 2013, 2016, 2019). Si bien este es un tema que ha venido despertando interés creciente en el país (Bellei, 2013; Valenzuela, Bellei y de los Ríos, 2014, entre otros) hasta ahora se ha abordado sin considerar la nacionalidad del estudiantado que, en el escenario descrito, adquiere relevancia.

\section{Factores que inciden en la segregación escolar}

La segregación escolar es un fenómeno complejo en el que concurren distintos elementos (Alegre, Benito y González, 2008; García Castaño y Olmos, 2012; Murillo y MartínezGarrido, 2018; Verger, Bonal y Zancajo, 2016).

Bellei (2013) distingue tres tipos de factores que se asociarían al fenómeno: de contexto, institucional y sociocultural. Dentro del primer tipo de factores, el más importante es la segregación residencial ${ }^{1}$ : si una ciudad se encuentra segregada en cuanto a cómo de distribuye su población en el territorio es probable que ese patrón se replique en composición de sus escuelas. En la segunda categoría, se incluirían todos los aspectos vinculados a la organización del sistema escolar, como la prerrogativa de algunos establecimientos de efectuar cobros a las familias, así como seleccionar o expulsar estudiantes. En la tercera categoría, se encontrarían las preferencias, estrategias y lógicas de elección de escuela de las familias.

\subsection{Factores de contexto}

En cuanto a la incidencia de la segregación residencial estudios efectuados en sistemas con políticas de zonificación documentan una asociación entre ésta y la segregación escolar (Alegre, Benito y González, 2008; Ong y Rickles, 2004; Taylor y Gorard, 2001). Para el caso español, en que sí existen políticas de zonificación, se ha documentado que escuelas de un mismo territorio y titularidad pueden exhibir proporciones muy desiguales de estudiantes extranjeros (Carrasco Pons 2011; García Castaño et al., 2012; Lubián, 2016; Peláez, 2012) y que la segregación escolar de este alumnado es superior a la segregación

\footnotetext{
${ }^{1}$ El concepto de segregación residencial tiene su origen en Estados Unidos en relación con la población afrodescendiente y migrante (Ruíz-Tagle, 2013). Ha sido posteriormente reinterpretado en otras latitudes con relación a las transformaciones urbanas de los últimos 30 años (Janochska, 2002). El concepto hace hincapié en el espacio que separa diferentes grupos que habitan las ciudades. Desde la definición de Sabatini, uno de los académicos chilenos que ha estudiado en mayor medida el fenómeno, la segregación residencial apunta al "grado de proximidad espacial o de aglomeración territorial de las familias pertenecientes a un mismo grupo social, sea que éste se defina en términos étnicos, etarios, de preferencias religiosas o socioeconómicos" (Sabatini, Cáceres y Cerda, 2001, p. 27).
} 
residencial de la población extranjera (López-Falcón y Bayona, 2012). Por su parte Kristen (2008) plantea para el caso alemán que el patrón residencial de familias migrantes no se ve reflejado en la distribución de los estudiantes migrantes entre las escuelas disponibles, mientras Rangvid (2007) sostiene que en Dinamarca, caracterizada por una segregación residencial de baja a moderada, se observan igualmente altos grados de segregación escolar étnica.

En Chile, país en que no existen políticas de zonificación, algunos autores asocian la concentración de estudiantes extranjeros en ciertas escuelas con el patrón residencial (Poblete 2016; Galaz, Poblete y Frías, 2017, por ejemplo), sin embargo, no tenemos noticia de estudios sistemáticos que lo documenten.

\subsection{Factores institucionales}

En relación a los factores institucionales, un amplio grupo de estudios en diversos países tales como Reino Unido (Ball, 1993, 1995; Ball, Maguire y Macrae, 1998; Tomlinson, 1997, 1998) o Francia (Broccolichi y Van Zanten, 2000, Van Zanten, 2003, 2015), sostienen que la presencia mayoritaria de minorías étnicas y migrantes en determinados centros educativos guarda estrecha relación con diversos aspectos de políticas educativas orientadas a la conformación de mercados escolares. Desde esta perspectiva las reformas de mercado en educación tienen importantes efectos en la reproducción de los privilegios y desventajas de las clases sociales y los grupos étnicos porque se alienta a la escuela a atender a los estudiantes con mejores ventajas de partida, y evitar a aquellos que pueden tener mayores dificultades en la adquisición de aprendizajes. No obstante, si bien la incorporación de este tipo de alumnado puede ser vista por gran parte de las escuelas como una amenaza, para otras puede representar una oportunidad, en perspectiva de lograr sobrevivir en un sistema altamente competitivo (Macrae, Maguire y Ball, 1998).

Chile es uno de los países que a nivel mundial ha implementado de forma más radical la conformación de un mercado educativo, lo que se encarna en diferentes políticas tales como: el financiamiento por alumno (voucher), la libre elección de escuela por parte de las familias, la amplia desregulación en el funcionamiento de las escuelas que ha caracterizado al sistema o el decidido apoyo al surgimiento de iniciativas privadas en el ámbito educativo (Bellei, 2015). En este contexto, los elementos que tendrían mayor influencia sobre una distribución desigual de estudiantes en función de sus características serían los cobros que efectúan algunos establecimientos por concepto de arancel o financiamiento compartido, así como sus prácticas de selección de estudiantes que han sido, hasta hace poco, abiertamente toleradas en el sistema escolar chileno.

Los estudios efectuados en Chile han destacado que, un factor clave para las familias extranjeras, es acceder a escuelas gratuitas, con vacantes disponibles y que faciliten los procesos de admisión (Stefoni et al., 2010; Joiko y Vásquez, 2016). Como ya se señaló, la mayor parte de los estudiantes extranjeros asisten a escuelas municipales caracterizadas por su gratuidad y escasos mecanismos de selección. Cabe destacar que este tipo de establecimiento ha experimentado una pérdida sostenida de estudiantes a partir de la década de los ' 80 , la que ha sido estimada en un $2 \%$ anual desde el año 2000 (Paredes y Pinto, 2009) y que se ha acompañado de un descenso de cerca de 1.000 escuelas de este tipo entre 1990 y 2016 (Cabezas e Irarrázaval, 2018). No obstante, la pérdida de matrícula en el sector municipal tiende a detenerse en los últimos años y esta estabilización se explicaría por la incorporación de estudiantes extranjeros en sus aulas (MINEDUC, 2017). 
Una dificultad extra para las familias extranjeras es que, hasta hace poco, cada escuela podía definir sus plazos y procedimientos de admisión (Joiko y Vásquez, 2016). La Ley de Inclusión Escolar, en vigor desde marzo de 2016, establece un nuevo sistema de admisión tanto para las escuelas municipales como privadas subvencionadas del país.

Junto a ello Galaz, Poblete y Frías (2017) documentan una serie de barreras informales que los migrantes deben sortear, para tener acceso a determinados servicios sociales, como el educativo. Una primera dificultad está representada por la exigencia de documentos nacionales de identidad, lo que ocurriría por desconocimiento de la normativa vigente y/o por la aplicación de criterios discrecionales del funcionario de turno, tendencia refrendada por UNESCO (2019) para el caso chileno. Asimismo, existirían barreras de tipo socio cultural, aludiendo con ello, a visiones estereotipadas sobre distintos grupos nacionales, especialmente afrodescendientes, así como la existencia de una "jerarquización de derechos", donde los ciudadanos chilenos tendrían prevalencia sobre los extranjeros en el acceso a los servicios sociales. Es más, los autores señalan que la concentración de estudiantes extranjeros en ciertas escuelas puede explicarse, además del patrón residencial de esta población, por el rechazo que algunos establecimientos hacen de este alumnado y su derivación a otros establecimientos que sí están dispuestos a efectuar los trámites necesarios para su matrícula. En esta línea el trabajo de Mardones (2006) plantea que el sistema escolar chileno se fomentaría una "incorporación guettizada" basada en una dinámica de exclusión/ inclusión en distintas escuelas: en algunas se niega el acceso por razones administrativas mientras en otras se produce una "sobre acogida".

\subsection{Factores socioculturales}

Las tendencias descritas para los factores institucionales se verían reforzadas por las acciones de los padres frente a la elección de escuela que formarían parte, en la categorización de Bellei (2013), de los factores de tipo sociocultural.

Existe un amplio cuerpo teórico que documenta que la libre elección de escuela favorece en mayor medida a las clases privilegiadas, teniendo los migrantes y minorías étnicas, menos información sobre el sistema escolar y, menos habilidades para interpretar ésta (Ball, 1993, 1995; Van Zanten, 2003, 2015). Para lograr tomar "buenas decisiones" en un mercado escolar, son fundamentales los capitales de tipo socioeconómico, cultural y social, con el que cuentan los electores más privilegiados, y de los que carecen los menos habilosos. Dichos recursos, definen el circuito de escolarización al cual acceden niños, niñas y jóvenes y, por tanto, su trayectoria escolar en el tiempo (Ball, Bowe y Gewirtz, 1996).

En términos generales, y en los sistemas en que existen políticas de zonificación, las clases populares y las minorías étnicas tienden a quedarse en las escuelas cercanas a sus domicilios, sin plantearse escolarizar a sus hijos en otras escuelas. Las elecciones de estas familias, son tomadas en función de aspectos prácticos en los que las condiciones del trabajo y la organización doméstica marcan importantes limitaciones, haciendo inviables ciertas opciones. Por su parte, las familias autóctonas tienden a evitar o abandonar las escuelas que concentran extranjeros: se trata del fenómeno de white flight documentado por diversos estudios (Broccolichi y Van Zanten, 2000; Fernández Enguita, 2008; Van Zanten, 1997).

Otro conjunto de investigaciones ha puesto el acento en el rol que las propias familias extranjeras o pertenecientes a minorías étnicas, pueden tener frente a la alta concentración 
de este tipo de alumnado en ciertas escuelas. Se apunta entonces, a prácticas de auto segregación. Diversos trabajos muestran que la observación de normas religiosas puede ser relevante en la elección de una escuela para los colectivos que profesan la religión islámica (Denessen, Driessena y Sleegers, 2005 para el neerlandés; Cebolla, 2007 para el caso británico; y Jamal Al-Deen, 2018, en el australiano). Por otra parte, Byrne y De Tona (2012) han documentado para el caso inglés que las familias extranjeras intentan equilibrar dos necesidades en su proceso de elección de escuela: por una parte, resguardar que sus hijos asistan a escuelas con otros semejantes a fin de evitar situaciones de racismo y, por otra, resistirse a las categorías raciales y de clase que puedan limitarlos (en ese sentido, una escuela racializada puede no ser la mejor opción).

Los estudios chilenos que abordan la elección de escuela por parte de familias extranjeras, sugieren que, además de lo mencionado en torno a coste y cercanía, para las familias migrantes, sería muy importante evitar situaciones de maltrato o discriminación. Por esta razón optarían por enviar a sus hijos a establecimientos donde se encuentren acompañados por familiares, amigos o connacionales (Stefoni et al., 2010; Joiko y Vásquez, 2016). Es decir, podría existir una opción consciente y voluntaria, por privilegiar escuelas con alta concentración de estudiantes extranjeros. Dicha opción, tiene relación con los hallazgos de diversos estudios que documentan que las escuelas chilenas son percibidas por niños extranjeros y sus familias como espacios hostiles, donde ocurren frecuentemente, situaciones de discriminación y racismo (Pavéz, 2012, 2013; Riedemann y Stefoni, 2015; Tijoux, 2013a, 2013b). Lo anterior, se ve respaldado por un estudio de la Superintendencia de Educación, que identifica a la discriminación por ser extranjero o por origen racial como uno de los ocho tipos de denuncias que más frecuentemente recibe este organismo (Superintendencia de Educación, 2017). Junto a ello un estudio de UNICEF destaca que un $20 \%$ de los niños chilenos piensa que los ciudadanos de otros países, como Perú, Bolivia o Haití, son inferiores a sus compatriotas (UNICEF, 2011).

Este trabajo presenta evidencia en torno a la incidencia de los factores socioculturales que podrían influir en la concentración de alumnado extranjero en ciertas escuelas. Las conclusiones respaldan el planteamiento de que los criterios y comportamientos de las familias extranjeras frente a la elección de una escuela para sus hijos, se orientan fuertemente por la búsqueda de un espacio protegido donde convivan con compatriotas que no los someterán a maltratos.

\section{Método}

Entre los años 2013 y 2016 se desarrolló una investigación centrada en el análisis de la segregación socioeconómica entre escuelas de enseñanza básica. Su diseño planteaba el estudio sincrónico de los tres elementos clave identificados por la literatura optándose por un estudio de casos con enfoque territorial. A través de la aplicación de Sistemas de Información Geográfica SIG se identificaron pequeños conjuntos de escuelas muy cercanas entre sí, los cuales se denominaron Unidad Geográfica Acotada UGA. Durante la investigación se estudiaron casos en Santiago, Valparaíso y Concepción (10, 4 y 5 casos respectivamente). Se asumió un enfoque metodológico mixto realizando, en una primera fase, entrevistas con madres y directivos y, en una segunda etapa, se aplicó una encuesta ad hoc. En algunas UGAS, especialmente interesantes, se efectuaron estudios etnográficos. Este trabajo presenta información producida en el marco de uno de ellos. 
El caso de estudio se ubica en una comuna de la zona centro de la ciudad de Santiago. Los indicadores socioeconómicos de la comuna la posicionan en una situación algo más desaventajada que el conjunto de la Región Metropolitana. En efecto, el porcentaje de personas en situación de pobreza por ingresos corresponde a $11,5 \%$, cifra que a nivel regional es de $9,2 \%$. Junto a ello, su proporción de población entre o y 14 años es menor $(17 \%)$ que la reportada para la Región y el país (20\%); asimismo, la proporción de adultos mayores (más de 65 años) resulta ser superior (13\% para el caso de la comuna y $10 \%$ para el de la región y el país) (BCN, 2018). Se destaca que se trata de una de las seis comunas de la Región Metropolitana que alberga una mayor proporción de migrantes (Ministerio del Interior y Seguridad Pública, 2017), predominando la nacionalidad peruana, seguida de la haitiana y colombiana.

Las dos escuelas municipales que componen el caso (y que denominaremos A y B) se ubican en una de las avenidas más importantes de la ciudad, contando con varios recorridos de autobuses y una estación de metro, lo que les proporciona gran conectividad con otros sectores de la ciudad. Las escuelas ocupan en conjunto casi la mitad de una manzana, una junto a la otra, separadas tan solo por una reja. Sus instalaciones fueron edificadas por la Sociedad Constructora de Establecimientos Educacionales hacia mediados del siglo Xx y se caracterizan por la amplitud de sus espacios, así como tres pisos en altura. Debido a esta similitud en su infraestructura, que es prácticamente continua, cuesta distinguir a simple vista que se trata de dos escuelas. El frontis de la escuela A se ubica en una calle pequeña, perpendicular a la gran avenida mencionada, mientras el de la escuela B da justo a esta gran arteria.

Ambos establecimientos fueron creados en el año 1928, como Escuela Superior de Hombres (Escuela A) y Escuela Superior de Mujeres (Escuela B), pero ninguna de ellas funcionaba en sus actuales edificios. Mientras la Escuela B ha mantenido sus características generales prácticamente inalteradas desde su creación, la Escuela $\mathrm{A}$ ha sufrido importantes modificaciones. En efecto, la Escuela B ha atendido siempre sólo a niñas en etapa de enseñanza básica y, posteriormente, también prebásica. El principal cambio que ha sufrido es la modificación en su nombre, pero en el barrio se le sigue conociendo por el original. Desde hace algunos años, se admite en prebásica a varones, sin embargo, éstos deben abandonar la escuela cuando pasan a primero básico, porque a partir de entonces sólo se atiende a niñas.

Como contrapunto, la escuela A ha tenido varias modificaciones relevantes a través del tiempo. Como se ha señalado, fue creada como establecimiento de enseñanza básica para varones, pero en el año 1989 su orientación cambia, incorporando desde entonces enseñanza media de tipo técnico profesional. Además, amplía su oferta atendiendo también a estudiantes de sexo femenino, manteniéndose su carácter mixto hasta hoy. Su nombre actual se le otorga en el año 2008 y, en los últimos años, se modifica también la modalidad de enseñanza media que otorga (pasando al régimen científico-humanista).

En relación a los resultados que las escuelas obtienen en Sistema de Medición de la Calidad de la Educación (SIMCE), se constata una tendencia sostenida donde la Escuela B muestra mejores rendimientos que la Escuela A. Ésta sólo se ha revertido en los últimos tres años para los cuales se dispone de datos (anexo 1).

En cuanto a la evolución de su matrícula y siguiendo la tendencia a nivel nacional, ambas escuelas municipales han visto mermado su número de estudiantes calculándose que entre el año 2004 y 2017 las escuelas A y B han perdido un 51\% y 52\% de matrícula 
respectivamente, a una disminución promedio anual del 5\% (anexo 2). Dicha tendencia sólo se ve interrumpida para ambos establecimientos en el año 2017 en que se observa una leve alza.

Finalmente, y este es el tema que concentra nuestra atención, ambas escuelas municipales escolarizan estudiantes extranjeros, pero la escuela A exhibe una proporción considerablemente superior a la escuela $\mathrm{B}$. El cuadro 1 sintetiza los datos de proporción de alumnado nacional y extranjero para los años 2015, 2016 y 2017 y, tal como se observa, la tasa de alumnado extranjero en la escuela A es siempre superior al de la escuela B por alrededor de 20 puntos porcentuales.

Cuadro 1. Proporción de estudiantes chilenos y extranjeros

\begin{tabular}{llcccc}
\hline & & \multicolumn{2}{c}{ ESCUELA A } & \multicolumn{2}{c}{ ESCUELA B } \\
\hline \multirow{2}{*}{2015} & Chilenos & 407 & $78,7 \%$ & 692 & $94,7 \%$ \\
& Extranjeros & 110 & $21,3 \%$ & 39 & $5,3 \%$ \\
\hline \multirow{2}{*}{2016} & Chilenos & 367 & $67,1 \%$ & 616 & $87,7 \%$ \\
& Extranjeros & 180 & $32,9 \%$ & 86 & $12,3 \%$ \\
\hline \multirow{2}{*}{2017} & Chilenos & 305 & $57,2 \%$ & 607 & $76,9 \%$ \\
& Extranjeros & 228 & $42,8 \%$ & 182 & $23,1 \%$ \\
\hline
\end{tabular}

Fuente: Elaboración propia sobre BBDD SIGE.

¿Cómo se puede explicar esta diferencia si se trata de escuelas municipales vecinas, ambas son gratuitas y no realizan procesos de selección de estudiantes? Este panorama despacharía la relevancia del patrón residencial de extranjeros y de los factores institucionales relativos a los costos y admisión como explicaciones plausibles. Por tanto, en principio, sólo restan los factores de tipo sociocultural para dar cuenta de esta diferencia.

En función de ello durante el año 2015 se desarrolló un estudio etnográfico por un período de siete meses. Durante el trabajo de campo se visitó de manera permanente el espacio público donde se ubican las dos escuelas, de tres a cuatro veces por semana, observando las dinámicas cotidianas de los estudiantes y familias. Para ello se utilizó una Pauta de observación etnográfica, orientada a tres focos: conformación del espacio, funcionamiento de las escuelas observable desde su exterior, comportamiento de estudiantes durante la entrada y salida de clases.

Asimismo, se desarrollaron entrevistas con 3 comerciantes que trabajan en las inmediaciones de los establecimientos atendiendo kioscos, 2 directivos y 22 madres y padres en ambas escuelas, 13 de ellos apoderados de la escuela A y 9 de la escuela B. En la escuela A se entrevistó a 6 chilenos/as y a 7 extranjeros (una venezolana y 6 peruanos/as). En la Escuela B se entrevistó a 6 chilenos/as y 3 extranjeros (una venezolana, una peruana y una dominicana). Las entrevistas fueron desarrolladas con padres, madres o apoderados que cumplieran alguna de las siguientes características: habían matriculado a sus hijos en una de las escuelas recientemente (como máximo un año), vivían a un kilómetro o menos de distancia o vivían a más de 3 kilómetros de distancia. Estas características eran relevantes para recoger información sobre los procesos de admisión y el peso de la distancia entre casa y escuela como factor de elección.

Las entrevistas, de tipo semi-estructuradas, se desarrollaron en torno a cuatro focos: razones a la base de la elección de escuela, características del proceso de admisión, existencia de cobros u otros requerimientos económicos por parte de la escuela y satisfacción con la elección. Éstas fueron grabadas e íntegramente transcritas, 
desarrollando posteriormente el proceso de codificación que permitió estructurar un análisis de contenido temático en base a ellas.

\section{Resultados}

Como se señaló antes, la primera explicación que algunos académicos están dando a la concentración de estudiantes extranjeros en determinadas escuelas alude al patrón residencial, es decir, se asume que los estudiantes extranjeros viven en las inmediaciones de estas escuelas. A través de las entrevistas realizadas se observa que existe disparidad en este aspecto, de manera que mientras algunos padres extranjeros señalan vivir muy cerca otros informan que su hogar se encuentra más alejado². Es relevante considerar que en Chile no existen políticas de zonificación escolar y diversas investigaciones recientes muestran que los estudiantes se desplazan por la ciudad recorriendo distancias variables para llegar a sus escuelas (Córdoba, Farris y Rojas, 2017; Donoso y Arias, 2013; Rodríguez et al., 2016).

Una segunda posible explicación se relaciona con los factores institucionales, dentro de los cuales resultan especialmente relevantes para el caso de escuelas chilenas tanto los cobros que pueden realizar éstas como los procesos de selección de su alumnado (ambos aspectos fueron recientemente regulados a través de la Ley de Inclusión en vigencia desde 2016). La información producida a través del estudio etnográfico indica que en ninguna de las escuelas que conforman la UGA existe selección del alumnado por rendimiento escolar, ni otras razones (situación económica, religión, nacionalidad). Tampoco se cobra mensualidad ni matrícula. Las familias informaron que cubren voluntariamente gastos menores asociados a la organización interna de los cursos para realizar paseos, festejar días importantes o comprar regalos.

La tercera explicación que podría estar interviniendo apunta a los factores socioculturales los que, tal como señalamos antes, guardan relación con las preferencias, comportamientos y/o estrategias de las familias frente a la elección de escuela. En esta línea, el trabajo de campo evidenció la existencia de notables diferencias en la valoración que existe en torno al prestigio académico y el alumnado de cada una de las escuelas.

\subsection{Prestigio y valoración de las escuelas}

En cuanto al prestigio académico, tal como ya se señaló, la Escuela B ha exhibido mejores resultados en el SIMCE que la posicionan en una posición privilegiada en relación a otros establecimientos del sector. Si bien los y las entrevistadas no aluden a las pruebas estandarizadas sí la perciben como una "buena" escuela en términos académicos ya que otorga una apropiada preparación para acceder a establecimientos públicos de enseñanza secundaria de gran prestigio, que aparece como la trayectoria anhelada para las hijas. De esta manera, la calidad académica de esta escuela representa una ventaja comparativa en relación a la escuela vecina percibida como de "menor nivel".

Encuentro que van bien, van un poquito adelantadas también y pretendo que con la educación que ella está teniendo acá pueda irse después a uno de esos liceos

\footnotetext{
${ }^{2}$ Este hallazgo coincide con los datos recabados a través de encuestas aplicadas en la primera fase de la investigación. Éstos muestran que la mitad de los estudiantes de ambas escuelas recorren una distancia aproximada igual o inferior a $1.5 \mathrm{kms}$., mientras la otra mitad efectúa trayectos superiores, no existiendo diferencias significativas entre ambas escuelas (anexo 3 ).
} 
emblemáticos de niña, como el Carmela (...) Ese es mi objetivo, por eso está acá. (Madre, Escuela B, chilena)

Mira este año yo lo vi en sexto, porque este año llegó una compañera que se crio en sala cuna y jardín con mi hija de sexto, pero ella estaba en el colegio de al lado. $Y$ si le afectó el cambio a la niña, de hecho, la niña está casi a punto de repetir porque tiene muy malas notas. $Y$ mi hija me cuenta y me dice: 'mamá es que ella no sabe nada...hay cosas que no le pasaron y a nosotras sí' me dice. (Madre, Escuela B, chilena)

Asimismo, se aprecia positivamente la formación actitudinal proporcionada por la escuela. Algunas madres señalan que todavía se mantiene una educación "a la antigua” haciendo referencia a la inculcación de valores o costumbres tradicionales como el correcto uso del uniforme escolar, el orden en la sala de clases o una actitud recatada por parte de las alumnas.

Es que encuentro que la educación... encuentro que es buena, o sea encuentro que como que tiene un modo bien antiguo de enseñar en este colegio. En todo, en la vestimenta porque todavía usan el jumper. Encuentro que eso es bueno, más tradicional, el delantal, todas esas cosas me gustan de ese colegio. (Madre, Escuela B, chilena)

El prestigio de la Escuela B se refuerza al ser considerada como una institución de "tradición familiar" en el sentido de que por generaciones las mujeres de una misma familia pueden haberse escolarizado allí.

Siempre tuve referencias buenas del colegio por tías que estudiaron ahí, mis primas estudiaron ahí, familiares, vecinos. Además, que toda mi familia es de (menciona un barrio), entonces muchos estudiaron acá; es como el conocido del sector. (Madre, Escuela B, chilena)

Todas estas características encarnan un ideal de "buena escuela” que también ha sido documentado por otros estudios (Córdoba, 2014; Hernández y Raczynski, 2015). La escuela A, por el contrario, y especialmente en el caso del alumnado de enseñanza media, carga con el estigma de ser la escuela que recibe "lo que botó la ola", es la escuela "de los porros", "de los desordenados", de los que por conducta fueron rechazados por otros establecimientos y "vinieron a parar aquí". Sus alumnos son percibidos como quienes se juntan en la plaza cercana a fumar marihuana o en la esquina a pelear con cuchillos. En algunas ocasiones estas riñas habrían requerido de la intervención de terceros, ya sea de carabineros o de personas que desde el autobús se han bajado para separarlos. La gente del sector evita involucrarse en estos conflictos, pues de hacerlo, según cuentan, la venganza de alguno de los grupos en conflicto es segura.

El año pasado por ejemplo llegaban fumando, estaban aquí afuerita fumando y botaban el pucho antes de entrar, o que el corte de pelo, o que las niñas llenas de maquillaje a más no poder asi y en cuanto a eso...porque yo me acuerdo que cuando llegó la directora un día se paró ahí y empezó: sacándole el gorro y que todos se sacaran la pintura y todo, pero antes no. O llegaban con la falda muy arriba y los pantys rotas, pero a propósito...entonces llegaban así, como que no era un colegio. (Madre, Escuela A, chilena)

Que en ese colegio fumaban marihuana, que andaban con cuchillo, que peleaban, que se violaban a los niños. Ese tipo de comentarios (...) No, nunca comprobé nada, pero si salían, no me acuerdo, parece que buscamos en internet y salían ahi los reclamos y todo el cuento. Igual daba como miedo porque él, chiquitito, recién empezando... (Madre, Escuela A, chilena)

... pero igual veo a los cabros cuando van entrando y no quisiera que él se rozara con esas personas (...) No, no me molesta que sean como sean, pero cuando se te contagia es difícil, bueno cada quien ve ahi (...) O sea, yo tenía compañeros que de repente iban con cuchillo, con machete, cosas así, que uno queda con la boca abierta. $Y$ bueno, yo tuve la suerte de que a mí nadie me molestó en el colegio, pero con gente así, ¿cómo 
lidias con los problemas que puede haber? No sé poh, que los papás eran traficantes o cosas así, que chuta, no querís eso cerca poh. (Madre, Escuela B, chilena)

Estos hallazgos vienen a refrendar lo ya documentado por diversos estudios chilenos en cuanto a la importancia que para las familias tiene el tipo de estudiante que asiste a las escuelas, llegándose a constituir como un criterio de calidad que orienta a las familias en cuanto a qué escuela es recomendable y cuál conviene evitar (Canales, Bellei y Orellana, 2016; Carrasco, Donoso y Mendoza, 2016; Córdoba, 2014; Falabella, Seppänen y Raczynski, 2015; Gubbins, 2014; Hernández y Raczynski, 2015; Rojas, Falabella y Leyton, $2015)$.

Además del comportamiento, existen tres elementos clave que distinguen claramente al alumnado de una y otra escuela: género, edad y nacionalidad.

La escuela B solo atiende a niñas de enseñanza básica, de manera que sus estudiantes son percibidas como "las niñitas". En este imaginario ellas se caracterizan por tener un mejor comportamiento, ser más tranquilas y ordenadas que los varones y, asimismo, su edad les hace ser vistas como inocentes. Estas características serían resguardadas por la Escuela $\mathrm{B}$, a través de los valores que cotidianamente se les transmitiría.

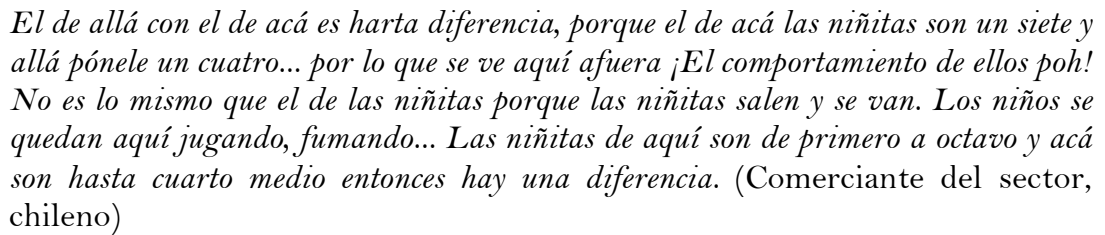

Por otra parte, a la Escuela A, que es mixta y atiende estudiantes tanto de enseñanza básica como media, se le atribuye un alumnado bastante más desordenado y rebelde: se trata de adolescentes, mujeres y hombres, muchos de los cuales habrían repetido una o varias veces, con lo cual en un mismo curso pueden convivir estudiantes con diferencias de dos o tres años de edad.

\begin{abstract}
Donde llegan muchas niñas a veces llegan niñas grandes, de más edad. Suponiendo ella tiene diez y hay una niña de trece y doce años. Entonces ellas hablan cosas que, por lo menos a mi no me gustaría que mi hija hablara, sobre sexualidad todavía. Que me pregunte mamá ¿̇qué significa este gesto? $\Upsilon$ ¿̇qué significa esto otro? Entonces yo como que esas cosas no me gustan (...) ¿̇Por qué las aceptaron?... no sé porque las aceptaron (risas), pero el colegio las aceptó. (Madre, Escuela A, chilena)

... quiero gente que tenga la misma edad de ella y que piensen como lo mismo porque mi hija todavía juega con muñecas, tiene una casa de muñecas, tiene un auto de muñecas, entonces ella todavía está en esa...ella todavía no pasa esa etapa de pololo, de que 'ah, quiero ver esto...', ella todavía es una niña y quiero que se mantenga así por un ratito porque después viene la adolescencia (...) Sí, porque las niñas ya piensan otras cosas. Supongamos las compañeras de ella ya se están dejando tocar, entonces eso no me gusta porque son chiquititas ìpara qué adelantarse tanto si después van a...pufff? Van a tener toda una vida para que les pase de todo ¿̇para qué se adelantan tanto? (Madre Escuela A, chilena)

Además de que yo veo a los chicos que salen de ese colegio y que se ponen a fumar marihuana afuera de mi colegio o en la esquina. No hay distinción entre grandes, chicos. Tú veí al hermano grande saliendo con el chico de primero, segundo básico de la mano y por el otro lado el pito. Nadie los controla tampoco, no hay carabineros, nada. (Madre Escuela A, chilena)
\end{abstract}

El tercer elemento que diferencia al alumnado de una y otra escuela es su nacionalidad. Como ya se señaló, la Escuela A atiende a una alta proporción de estudiantes migrantes y su ingreso masivo ha contribuido a fortalecer la imagen negativa de este establecimiento, 
debido a que se les percibe como desordenados, conflictivos o, más radicalmente, como delincuentes. Tal como han documentado otros estudios, en Chile este estigma se vincula fuertemente con lo indígena o lo negro (Tijoux, 2016).

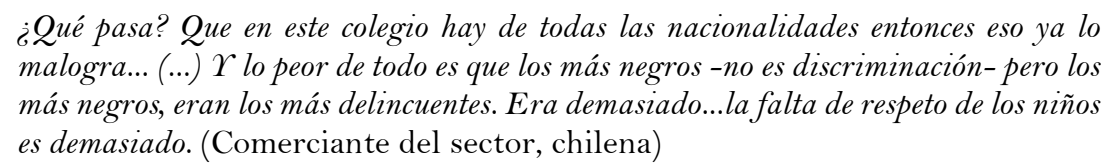

\subsection{Los criterios de elección de las familias nativas y extranjeras}

Como hemos mostrado, la escuela A se encuentra fuertemente desvalorizada en función de las características de su alumnado. Cabe entonces la pregunta respecto de cuáles son las razones que han tenido los padres -nativos y migrantes- para escolarizar a sus hijos allí. En el caso de los primeros, se confirma lo ya señalado por estudios previos: sus opciones se han visto limitadas por sus recursos económicos, han otorgado relevancia a la cercanía entre la escuela y la casa, el lugar de trabajo o de estudios, o bien, tenían buenas referencias de la escuela (Córdoba, 2014; Hernández y Raczynski, 2015). Sin embargo, las madres chilenas entrevistadas tienden a sentirse insatisfechas con su elección y varias barajaban la posibilidad de cambiar a sus hijos lo antes posible de escuela. Esta insatisfacción se vincula a diversos elementos. Varias de ellas señalan que les desagrada que la escuela acepte a todos los estudiantes que solicitan matrícula, manifestando explícitamente su expectativa de que se seleccione a los estudiantes en función de su comportamiento. Asimismo, expresaron su desacuerdo con que niños pequeños convivan con estudiantes repetidores.

... igual me gustaría que seleccionaran más a los niños porque como que todos dicen
que todos los que votan de otros colegios, que son desordenados a más no poder los
reciben acá (...) porque, por ejemplo, en el curso de la Janina hay compañeritos que
son, pero igroseros, pero a más no poder! Entonces no sé poh, igual perjudican a los
demás niños que se concentran. (Madre, Escuela A, chilena)
Yo le voy a ser bien franca, el liceo a mí no me gusta. No me gusta por ningún motivo.
Si tuviera otro lugar, yo las saco y las pongo, pero no puedo en este minuto (...) Que
era malo me decían, que este liceo no era pa' las niñas, que era malo, que era peligroso
pa' las niñas pequeñas. Pero yo las dejé, como están juntas, y ellas se cuidan. La más
grande es súper protectora de su hermanita. Así que yo sé que se van a cuidar, espero
no lamentarlo algún día porque estas cosas cuando pasan uno dice jchuta!... (Madre,
Escuela A, chilena)

La insatisfacción de las madres entrevistadas con esta escuela no se circunscribe únicamente a las características del alumnado. Existe una apreciación negativa de la gestión que, desde la nueva Dirección del establecimiento, se hace del comportamiento de los estudiantes y de su relación con las familias. En algunos casos, los entrevistados valoran positivamente que esta autoridad haya asumido una actitud más estricta frente a los estudiantes, pero otros consideran que se ha incurrido en maltrato tanto hacia ellos como hacia los apoderados. Asimismo, todos los entrevistados criticaron la nueva medida de limitar la entrada de madres, padres y apoderados al establecimiento: en el pasado no existían restricciones al respecto, posteriormente sólo se les permitía entrar hasta el patio de recepción lo que les genera inseguridad y desconfianza.

En el caso de las familias extranjeras la escasez de recursos económicos y la relativa cercanía con la casa también explican la opción por este establecimiento. Junto a ello se confirma lo señalado por otros estudios respecto a que para estas familias resulta clave el que la escuela facilite los procedimientos de admisión (Joiko y Vásquez, 2016; Stefoni et 
al., 2010). Todas las entrevistadas informaron que en la escuela A se encontraron con funcionarios que facilitaron la escolarización de sus hijos, lo que no necesariamente ocurre en otras escuelas, aun cuando sean municipales y se ubiquen en la misma comuna. Una de nuestras entrevistadas informó que en la primera escuela municipal de la comuna a la que se acercó para matricular a sus hijos, y que quedaba aún más cerca de su hogar, tuvo un recibimiento muy poco acogedor, confirmándose así lo señalado por otros estudios (Galaz, Poblete y Frías, 2017):

\begin{abstract}
Bueno, este... yo vine acá al colegio (menciona otra escuela municipal de la comuna), en el colegio este, me hicieron como a un lado se puede decir, me hicieron esperar por gusto como una media hora. Yo andaba con mis tres niños, pero después me dejaron allá afuera y ni siquiera me dejaron pasar, me atendieron de afuera, entonces, vi que no hacían nada. Después volví a tocar el timbre, me dijo que no. Entonces yo me sentí muy mal sinceramente. (Madre, Escuela A, peruana)
\end{abstract}

Junto a ello nuestros hallazgos indican que las madres y padres entrevistados privilegian un espacio escolar protegido para sus hijos en un escenario percibido como hostil. Ellos y ellas manifiestan expresamente la preocupación de que sus hijos vivan experiencias negativas o sean víctimas de bullying por parte de sus compañeros.

En la Escuela A esta situación podría evitarse, en gran medida, por dos razones. En primer lugar, allí ya existe un alto porcentaje de alumnos extranjeros. Por otra parte, esta escuela ofrece la posibilidad de escolarizar en el mismo espacio a niños y niñas, tanto en enseñanza básica como media, lo que resulta ser un elemento clave. El hecho de que sus hijos o hijas cuenten en la escuela con un hermano/a, primo/a, amigo/a o incluso un vecino/a en la misma escuela les proporciona a las familias una sensación de seguridad, porque habrá alguien que pueda acompañar a los niños en su proceso de adaptación a la nueva escuela y se una a ellos en caso de que sea necesario defenderse.

Yo, bueno, lo que pensaba, generalmente, por lo que él es de otro país, a veces, los maltratan, entonces, ese era mi entorno, buscarle un espacio donde él esté seguro y donde no le hagan diferencias, más que todo. (Madre, Escuela A, peruana)

Entonces que los dos se cuidan en el colegio por eso que no opté por ponerla en el colegio de niñas (...) Lo pensé, pero como te digo, yo siempre a mis niños los he tenido juntos en el colegio y como que se cuidan, como que se protegen. Entonces si ponía a Gisela en el colegio de niñas no iba a ver a su hermano (...) Sí, tenía unos sobrinos estudiando ahí. Me decían que es bueno, buena la enseñanza, pero... no pues... me importa más que se cuiden mis niños (...) Al principio le chocó, que no quería estudiar, que lloraba porque no quería venir al colegio. To le decía 'Pero ¿̇por qué no quieres venir al colegio?', 'No, por nada', hasta que después como que reaccionó mal en el colegio y la tía que estaba me mandó a llamar: 'Que sí, que Darío es un mal criado, que reaccionó así, que tiró a silla'. Entonces yo agarré le dije: 'No, pasa algo, ¿¿Darío, ¿qué paso?', 'No, que me pegan', que lo insultaban, que le decían garabatos. Entonces agarré yo, él estaba en $2^{\circ}$ y un niñito de $4^{\circ}$ le había pegado (...) A Darío lo molestaban porque era peruano, porque recién había venido y le pegaban pues. $\Upsilon$ la tía también como que no les tenía paciencia. (Madre, Escuela A, peruana)

... la verdad porque tenía un vecino que me aconsejó porque aquí había como que más este... más este... alumnos de afuera, extranjeros. Entonces era por algo que yo había conversado con el vecino y me dijo: 'no, no hay ningún problema de la cuestión de bullying no nada'. Claro porque al principio también lo mismo, pensaba que iba a ser chocante para ellos (...) Lo único que yo les anticipaba: te molesta a la primera, déjala pasar; te molesta a la segunda, avisale al profesor; a la tercera no te hace caso ni el profesor, ni el mismo y a la cuarta... si no tampoco lo van agarrar como dicen por débil o por inocente también pues. (Madre, Escuela A, peruana) 
Las motivaciones de las familias migrantes para optar por la Escuela A son claramente sintetizadas por la Directora de la escuela vecina que, como hemos señalado, acoge a una proporción menor de este alumnado:

Que es mixto yo creo y tiene enseñanza media, entonces tiene una mayor carta de alumnas y alumnos que pueden ingresar. Nosotros estamos sectorizados dentro de párvulo y hasta $8^{\circ}$ básico nada más y más encima puras niñas. Por lo tanto, te entra menos (...) O apoderados que prefieren que sus hijos estén juntos, entonces ponen a ambos ahí. (Directora, Escuela B)

\section{Conclusiones}

La concentración de estudiantes extranjeros o de minorías étnicas en determinadas escuelas es un fenómeno observado en diferentes latitudes que comienza a constatarse también en el caso chileno, asociado a la incorporación creciente de estudiantes extranjeros en el sistema escolar.

Habitualmente la concentración de este alumnado en ciertas escuelas es explicada en función del patrón residencial. El caso presentado pone en cuestión esta hipótesis: al tratarse de una dupla de escuelas vecinas lo esperable desde esta perspectiva habría sido encontrar proporciones similares de alumnado ex tranjero en ambas, lo que no ocurre. Esto es así a pesar de que los dos establecimientos son municipales y, por tanto, establecen menos barreras de entradas relativas a costo y selección que establecimientos privados. Paralelamente otras investigaciones han documentado que los estudiantes extranjeros asisten con mayor frecuencia a escuelas municipales, sin embargo, el caso presentado pone en evidencia que, dentro de un mismo territorio y teniendo la misma dependencia, las escuelas pueden no escolarizarlos en similares proporciones. Se confirma así una incorporación guettizada (Mardones, 2006) incluso dentro de la red pública o municipal: mientras algunas escuelas rechazan al estudiantado inmigrante, otras lo acogen y facilitan su escolarización.

Nuestros hallazgos sugieren que, para el caso estudiado, el factor sociocultural es aquel que parece proporcionar una mejor explicación para comprender por qué los estudiantes extranjeros asisten en mayor medida a una escuela municipal que a otra. Esto ocurriría en relación a dos elementos.

En primer término, las escuelas estudiadas son valoradas de forma diferente en función de las características de su alumnado. En un extremo se encuentra la escuela de las niñitas, a quienes se asocia con características positivas como adecuado rendimiento escolar, buen comportamiento e inocencia. En el otro se encuentra la escuela vecina que trabaja con niños, niñas y adolescentes, que pueden haber sido expulsados de otros establecimientos. Existe, además, un menor grado de satisfacción por parte de las familias chilenas quienes se plantean seriamente cambiar a sus hijos de escuela. Este deseo no tiene que ver exclusivamente con el comportamiento de su alumnado, sino con factores relativos a la gestión directiva de establecimiento. Se trata entonces de una escuela que concentra dificultades y se posiciona en un lugar de menor valoración social que el establecimiento vecino. Es esta escuela -la de los porros- la que termina escolarizando en mayor medida a los estudiantes extranjeros.

Sin embargo, no parece ser que la llegada de este nuevo alumnado explique, por sí misma, la visión negativa que existe acerca de la escuela. Esto, al parecer, sólo viene a reforzar un proceso de desvalorización previo. Se trataría de una tendencia similar a la documentada 
por estudios relativos a barrios que albergan altas proporciones de población extranjera en el sentido que éstos ya se encontraban en un proceso de degradación anterior a la llegada de esta población (Aramburu, 2001).

En segundo término, se constata que las familias extranjeras se orientan en mayor medida hacia una de las escuelas con el fin de proteger a sus hijos. Tal como ha sido señalado por otros estudios (Joiko y Vásquez, 2016; Stefoni, 2010), uno de los principales criterios que estarían poniendo en juego las familias extranjeras en la elección de una escuela en Chile es evitar que sus hijos sean víctimas de bullying o maltrato por parte de sus compañeros. Las familias entrevistadas conocían de cerca esta situación, ya sea porque ellos mismos la habían experimentado o porque personas cercanas lo habían hecho. Es interesante destacar que estas familias parecen estar dispuestas a sacrificar otros criterios de elección de escuela, como "calidad académica", en función de ofrecer a sus hijos un espacio escolar tranquilo y acogedor. Desde el punto de vista de estas familias este tipo de escuela, que de alguna manera tiende a especializarse en la atención al alumnado extranjero, puede ser positivamente valorada porque responde a una legítima preocupación por el bienestar de sus hijos, pero representan a la vez una nueva forma de segregación escolar.

Los hallazgos presentados indican que la concentración de alumnado extranjero sí se vincula en este caso con características institucionales relativas a la oferta educativa de los establecimientos. Dado que las familias extranjeras privilegian la tranquilidad de sus hijos en el espacio escolar, ciertas características de éstas (como si reciben a estudiantes de ambos sexos o si se ofertan más niveles educativos) no son superfluas y facilitarían la concentración de este tipo de alumnado en unas escuelas y no en otras. Mientras más amplia sea la oferta de la escuela, más posibilidades hay de que un estudiante extranjero pueda contar con un paisano (del mismo u otro sexo, en el mismo u otro nivel escolar) que le acompañe, proteja y eventualmente defienda. De esta forma, muy probablemente si las dos escuelas estudiadas fuesen idénticas en su oferta, niños y niñas extranjeros se distribuirían de forma más equitativa en ambas.

A partir de la Ley de Inclusión Escolar (2015) se están implementando importantes transformaciones en el proceso de admisión a las escuelas del país. Se está pasando de una situación en que básicamente no existían regulaciones (tolerándose la selección de estudiantes basada en su rendimiento real o potencial) a otra en que se establece un sistema de admisión único para escuelas sostenidas con fondos públicos (Sistema de Admisión Escolar-SAE) en que ya no pueden seleccionar a su alumnado por razones académicas, socioeconómicas, culturales o étnicas. Este sistema garantiza que las familias puedan escoger la escuela de sus hijos, presentando postulaciones a varias opciones definidas por ellas mismas. Asimismo, la ley busca poner fin al financiamiento compartido no existiendo, sin embargo, una fecha límite para ello. El sentido de ambas regulaciones es debilitar las barreras de entrada que tradicionalmente habían impuesto las escuelas a las familias y propender a mayores grados de integración social.

El análisis del funcionamiento del SAE muestra que los estudiantes prioritarios no habrían variado su postulación a escuelas municipales y privadas subvencionadas (lo que se relacionaría con la vigencia del sistema de financiamiento compartido) y su distribución entre establecimientos es similar a la que se constataba previo a la aplicación del nuevo sistema (Sillard, Garay y Troncoso, 2018). Junto a ello se observa estabilidad en la brecha que existe entre la postulación de estudiantes prioritarios y no prioritarios a escuelas calificadas como "deseables", aun cuando existen leves incrementos en la postulación a 
este tipo de escuela, tanto por parte de estudiantes prioritarios como no prioritarios (Carrasco y Honey, 2019). Esta tendencia a la estabilidad se explicaría tanto por las opciones que hacen las familias prioritarias frente a la postulación, decantándose por escuelas de menor calidad, con menos demanda e históricamente menos selectivas (Carrasco y Honey, 2019), como por la "oferta" educativa que efectivamente tienen a su alcance en los territorios en que residen (Carrasco et al., 2019).

A la luz de estos resultados preliminares y de los hallazgos mostrados en este trabajo, probablemente las familias inmigrantes continúen optando por establecimientos que concentran este tipo de estudiantes, a pesar de la existencia de nuevas regulaciones que, en teoría, podrían abrirles otras posibilidades.

\section{Agradecimientos}

Este trabajo forma parte del Proyecto FONDECYT de Iniciación a la Investigación N${ }^{\circ} 1130149$ Análisis de la segregación escolar socioeconómica en Enseñanza Básica. Se agradece el apoyo del Programa FONDECYT.

\section{Referencias}

Alegre, M., Benito, R. y González, I. (2008). Procesos de segregación y polarización escolar: La incidencia de las políticas de zonificación escolar. Profesorado. Revista de Curriculum y Formación del Profesorado, 12(5), art 5.

Aramburu, M. (2001). El mito de la huida autóctona. El caso de Ciutat Vella, Barcelona. Scripta Nova Revista Electrónica de Geografía y Ciencias Sociales, 5, 38-54. https://doi.org/10.1344/sn2001.5.388

Ball, S. (1993). Education markets, choice and social class: The market as a class strategy in the UK and the USA. British Journal of Sociology of Education, 14(1), 3-19. https://doi.org/10.1080/0142569930140101

Ball, S. (1995). Circuits of schooling: A sociological exploration of parental choice of school in social class context. Sociological Review, 43(1), 52-78. https://doi.org/10.1111/j.1467-954X.1995.tbo2478.x

Ball, S., Bowe, R. y Gewirtz, S. (1996). School choice, social class and distinction: The realization of social advantage in education. Journal of Education Policy, 11(1), 89-112. https://doi.org/10.1080/0268093960110105

Ball, S., Maguire, M. y Macrae, S. (1998). Race, space and the further education market place. Race Ethnicity and Education, 1(2), 171-189. https://doi.org/10.1080/1361332980010203

Bellei, C. (2013). El estudio de la segregación socioeconómica y académica de la educación chilena. Estudios Pedagógicos, 39(1), 325-345. https://doi.org/10.4067/S07 18-07052013000100019

Bellei, C. (2015). El gran experimento. Mercado y privatización de la educación chilena. Santiago de Chile: LOM.

BCN. (2018). Reportes comunales 2015. Recuperado de https://reportescomunales.bcn.cl/2015/index.php/Categor\%C3\%ADa:Comunas

Broccolichi, S. y Van Zanten, A. (2000). School competition and pupil flight in the urban periphery. Journal Education Policy, 15(1), 51-60. https://doi.org/10.1080/026809300286015 
Byrne, B. y De Tona, C. (2012). Trying to find the extra choices: Migrant parents and secondary school choice in greater Manchester. British Journal of Sociology of Education, 33(1), 21-39. https://doi.org/10.1080/01425692.2012.632865

Cabezas, V. y Irarrázaval, I. (2018). Des municipalización y la nueva educación pública. En I. Sánchez (Ed.), Ideas en educación II. Definiciones en tiempos de cambio (pp. 223-254). Santiago de Chile: Ediciones UC.

Canales, M., Bellei, C. y Orellana, V. (2016). ¿Por qué elegir una escuela privada subvencionada? Sectores medios emergentes y elección de escuela en un sistema de mercado. Estudios Pedagógicos, 42(3), 89-109. https://doi.org/10.4067/S07 18-07052016000400005

Carrasco, A. y Honey, N. (2019). Nuevo sistema de admisión escolar y su capacidad de atenuar la desigualdad de acceso a colegios de calidad: Al inicio de un largo camino. Santiago de Chile: Carrasco, A., Donoso, A. y Mendoza, M. (2016). La dimensión ético-política de la elección de escuela: Dilemas en familias chilenas de elite. En J. Corvalán, A. Carrasco, y J. E. García Huidobro (Eds.), Mercado escolar y oportunidad educacional. Libertad, diversidad y desigualdad (301-335). Santiago de Chile: Ediciones UC.

Universidad Católica de Chile.

Carrasco, A., Honey, N., Oyarzún, J. y Bonilla, A. (2019). Nuevo sistema de admisión escolar ¿Conviene reformarlo? En A. Carrasco y L. Flores (Eds.), De la reforma a la transformación. Capacidades, innovaciones y regulación de la educación chilena (pp. 423-446). Santiago de Chile: Ediciones Universidad Católica de Chile.

Carrasco Pons, S. (2011). Segregación escolar e inmigración: Repensando planteamientos y alternativas. En F. García Castaño y S. Carrasco Pons (Eds.), Población inmigrante y escuela: Conocimientos y saberes de investigación (pp. 269-287). Barcelona: Ministerio de Educación.

CASEN. (2017). Inmigrantes. Síntesis de resultados. http://observatorio.ministeriodesarrollosocial.gob.cl/casenmultidimensional/casen/docs/Resultados_Inmigrantes_casen_2017.pdf

Castillo, D., Santa Cruz, E. y Vega, A. (2018). Estudiantes migrantes en escuelas públicas chilenas. Calidad en la Educación, 49, 18-49. https://doi.org/10.31619/caledu.n49.575

Cebolla, H. (2007). La concentración de minorías étnicas en las escuelas británicas: Un análisis sobre la elección de centros. Revista Española de Investigaciones Sociológicas, 118, 97-121.

Córdoba, C. (2014). La elección de escuela en sectores pobres. Resultados de un estudio cualitativo. Psicoperspectivas, 13(1), 56-67. https://doi.org/10.5027/psicoperspectivas-Vol13-Issue1fulltext-301

Córdoba, C. y Miranda, P. (2018). Incorporación de estudiantes migrantes en el sistema escolar chileno: ¿Una nueva forma de segregación escolar? Contribuciones Científicas y Tecnológicas, $43(1), 43-54$.

Córdoba, C., Farris, M. y Rojas, K. (2017), Discussing school socioeconomic segregation in territorial terms: The differentiated influence of urban fragmentation and daily mobility. Investigaciones Geográficas, 92, 47-66.

Denessen, E., Driessena, G. y Sleegers, P. (2005). Segregation by choice? A study of group-specific reasons for school choice. Journal of Education Policy, 20(3), 347-368.

https://doi.org/10.1080/02680930500108981

Donoso, S. y Arias, O. (2013). Desplazamiento cotidiano de estudiantes entre comunas de Chile: Evidencia y recomendaciones de política para la nueva institucionalidad de la Educación Pública. EURE, 39(116), 39-73. https://doi.org/10.4067/S0250-71612013000100002 
Falabella, A., Seppänen, P. y Raczynski, D. (2015). Growing tolerance of pupil selection: Parental discourses and exclusionary practices in Chile and Finlandia. En P. Seppänen, A. Carrasco, M. Kalalahti, R. Rinne y H. Simola (Eds.), Contrasting dynamics in education politics of extremes. School choice in Chile and Finland (pp. 121-138). Rotterdam: Sense Publishers.

Fernández Enguita, M. (2008). Escuela pública y privada en España: La segregación rampante. Revista de la Asociación de Sociología de la Educación, 1(2), 42-69.

Galaz, C., Poblete, R. y Frías, C. (2017). Las operaciones de exclusión de personas inmigradas a través de las políticas públicas en Chile. Revista del CLAD Reforma y Democracia, 68, 169204.

García Castaño, F. y Olmos, A. (2012). Segregaciones y construcción de la diferencia en la escuela. Madrid: Trotta.

García Castaño, F., Rubio, M., Olmos, A. y López, R. (2012). Todos lo sabían...naturalización de los procesos de agrupación y segregación escolar en un barrio andaluz. En F. García Castaño y A. Olmos (Eds.), Segregaciones y construcción de la diferencia en la escuela (pp. 83118). Madrid: Trotta.

Gubbins, V. (2014). Estrategias educativas de familias de clase alta. Un estudio exploratorio. Revista Mexicana de Investigación Educativa, 19(63), 1069-1089.

Hernández, M. y Raczynski, D. (2015). Elección de escuela en Chile. De las dinámicas de distinción y exclusión a la segregación socioeconómica del sistema escolar. Estudios Pedagógicos, 41(2), 127-141. https://doi.org/10.4067/S07 18-07052015000200008

INE-DEM. (2018). Estimación de personas extranjeras residentes en Chile al 31 de diciembre de 2018. Informe Metodológico. Recuperado de

https://www.extranjeria.gob.cl/media/2019/07/Estimaci\%C3\%B3n-Poblaci\%C3\%B3nExtranjera-en-Chile.pdf

Jamal Al-Deen, T. (2018). Class, honour and reputation: Gendered school choice practices in a migrant community. The Australian Educational Researcher, 45, 401-417. https://doi.org/10.1007/s 13384-017-0255-6

Janoschka, M. (2002). El nuevo modelo de la ciudad latinoamericana: Fragmentación y privatización. EURE, 28(85), 11-20. https://doi.org/10.4067/S0718-45652016000200005

Joiko, S. y Vásquez, A. (2016). Acceso y elección escolar de familias migrantes en Chile: No tuve problemas porque la escuela es abierta, porque acepta muchas nacionalidades. Calidad en la Educación, 45, 132-173.

Kristen, C. (2008). Primary school choice and ethnic school segregation in german elementary schools. European Sociological Review, 24(4), 495-510. https://doi.org/10.1093/esr/jcno15

López-Falcón, D. y Bayona, J. (2012). Segregación residencial en Barcelona: Del boom migratorio al asentamiento. En F. García Castaño y A. Olmos (Eds.), Segregaciones y construcción de la diferencia en la escuela (pp. 21-41). Madrid: Trotta.

Lubián, C. (2016). La escolarización del alumnado de nacionalidad extranjera en la ciudad de Granada. Diferencias por nacionalidad y el papel de la red privada-concertada. Revista de la Asociación de Sociología de la Educación, 9(2), 212-231.

Macrae, S., Maguire, M. y Ball, S. (1998). Competition, choice and hierarchy in a post-16 market. En S. Tomlinson (Ed.), Education 14-19. Critical perspectives (pp. 88-100). Londres: The Athlone Press.

Mardones, P. (2006). Exclusión y sobre-concentración de la población escolar migrante bajo un modelo de segregación socio-territorial. Recuperado de http://bibliotecavirtual.clacso.org.ar/ar/libros/becas/2005/2005/migra/mardones.pdf 
Margarit, D. y Galaz, C. (2018). Espacios barriales y convivencia: Reflexiones sobre las concentraciones de población inmigrada y la territorialidad urbana. Rumbos TS, 17, 23-50.

MINEDUC. (2017). Minuta variación de matrícula 2017 por dependencia, región y comuna. Recuperado de http://participacionciudadana.mineduc.cl/difusion-y-estudios/

MINEDUC. (2018). Política nacional de estudiantes extranjeros 2018-2022. Recuperado de https://migrantes.mineduc.cl/wp-content/uploads/sites/88/2018/06/POLITICANACIONAL-EE-Final-1-1.pdf

MINEDUC. (2019). Minuta de variación de matrícula preliminar. Minuta 06. Recuperado de https://centroestudios.mineduc.cl/wp-content/uploads/sites/100/2019/08/MINUTA6_MatriculaPreliminar.pdf

Murillo, F. y Martínez-Garrido, C. (2018). Magnitud de la segregación escolar por nivel socioeconómico en España y sus Comunidades Autónomas y comparación con los países de la Unión Europea. Revista de la Asociación de Sociología de la Educación, 11(1), 37-58. https://doi.org/10.7203/RASE.11.1.10129

Ong, P. y Rickles, J. (2004). The continued nexus between school and residential segregation. Berkeley La Raza Law Journal, 15(1), 25-45.

OECD. (2013). PISA 2012 results. Excellence through equity. París: OECD Publishing.

OECD. (2016). PISA 2015 results. Excellence and equity in education. París: OECD Publishing.

OECD. (2019). PISA 2018 results. Where all students can succeed. París: OECD Publishing.

Paredes, R. y Pinto, J. (2009). ¿El fin de la educación pública en Chile? Estudios de Economía, 36(1), 47-66. https://doi.org/10.4067/S07 18-52862009000100003

Pavéz, I. (2012). Inmigración y racismo: Experiencias de la niñez peruana en Santiago de Chile. Si Somos Americanos, 12(1), 75-99. https://doi.org/10.4067/So7 19-09482012000100004

Pavéz, I. (2013). Los significados de ser niña y niño migrante: Conceptualizaciones desde la infancia peruana en Chile. Polis, 12(35), 1-19. https://doi.org/10.4067/So7 18-65682013000200009

Peláez, C. (2012). La escuela, un espacio simbólico que construir: Estigmas y estrategias de los agentes en los procesos de segregación étnica y escolarización. En F. García Castaño y A. Olmos (Eds.), Segregaciones y construcción de la diferencia en la escuela (pp. 61-81). Madrid: Trotta.

Poblete, R. (2016). Niños y niñas migrantes: Trayectorias de inclusión educativa en escuelas de la región metropolitana. Recuperado de https://www.supereduc.cl/wpcontent/uploads/2017/01/Informe-Final-de-Investigacio\%CC\%81n-Migrantes.pdf

Rangvid, B. (2007). Living and learning separately? Ethnic segregation of school children in Copenhagen. Urban Studies, 44(7), 1329-1354.

https://doi.org/10.1080/00420980701302338

Riedemann, A. y Stefoni, C. (2015). Sobre el racismo, su negación y las consecuencias para una educación anti-racista en la enseñanza secundaria chilena. Polis, 14(42), 1-18. https://doi.org/10.4067/SO7 18-65682015000300010

Rodríguez, P., Truffello, R., Suchan, K., Varela, F., Matas, M., Mondaca, J., Céspedes, J., Valenzuela, L., Valenzuela, J. P. y Allende, C. (2016). Apoyando la formulación de políticas públicas y toma de decisiones en educación utilizando técnicas de análisis de datos masivos. Santiago de Chile: Universidad de Chile.

Rojas, N. y Silva, C. (2016). La migración en Chile. Breve reporte y caracterización. Recuperado de https://www.extranjeria.gob.cl/media/2016/08/informe_julio_agosto_2016.pdf 
Rojas, M. T., Falabella, A. y Leyton, D. (2016). Madres de clase media frente al mercado educativo en Chile: Decisiones y dilemas. En J. Corvalán, A. Carrasco y J. E. García Huidobro (Eds.), Mercado escolar y oportunidad educacional. Libertad, diversidad y desigualdad (pp. 233-267). Santiago de Chile: CEPPE.

Ruiz Tagle, J. (2013). A theory of socio-spatial integration: Problems, policies and concepts from a US perspective. International Journal of Urban and Regional Research, 37(2), 388-408. https://doi.org/10.1111/j.1468-2427.2012.01180.x

Sabatini, F., Cáceres, G. y Cerda, J. (2001). Segregación residencial en las principales ciudades chilenas: Tendencias de las tres últimas décadas y posibles cursos de acción, EURE, 28(82), 21-42. https://doi.org/10.4067/S0250-71612001008200002

Sánchez, K., Valderas, J., Messenger, K., Sánchez, C. y Barrera, F. (2018). Haití, la nueva comunidad inmigrante en Chile. Revista Chilena de Pediatría, 89(2), 278-283. https://doi.org/10.4067/So370-41062018000200278

Sillard, M., Garay M. y Troncoso, I. (2018). Análisis al nuevo sistema de admisión escolar en Chile. La Región de Magallanes como experiencia piloto. Calidad de la Educación, 49, 112-136. https://doi.org/10.31619/caledu.n49.578

Stefoni, C., Acosta, E., Gaymer, M. y Casas-Cordero, F. (2010). Niños y niñas migrantes en Santiago de Chile. Entre la integración y la exclusión. Santiago de Chile: OIM-Universidad Alberto

Superintendencia de Educación. (2017). Discriminación en la escuela. Descripción y análisis a partir de las denuncias recibidas por la superintendencia de Educación. Recuperado de https://www.supereduc.cl/

Taylor, C. y Gorard, S. (2001). The role of residence in school segregation: Placing the impact of parental choice in perspective. Environment and Planning, 33, 1829-1852. https://doi.org/10.1068/a34123

Tijoux, M. E. (2013a). Las escuelas de la inmigración en la ciudad de Santiago: Elementos para una educación contra el racismo. Polis, 12(35), 287-307. https://doi.org/10.4067/So71865682013000200013

Tijoux, M. E. (2013b). Niños(as) marcados por la inmigración peruana: Estigma, sufrimientos, resistencias. Convergencia, 20(61), 83-104.

Tijoux, M. E. (2016). Racismo en Chile. La piel como marca de la inmigración. Santiago de Chile: Editorial Universitaria.

Tomlinson, S. (1997). Diversity, choice and ethnicity: The effects of educational markets on ethnic minorities. Oxford Review of Education, 23(1), 63-76. https://doi.org/10.1080/0305498970230106

Tomlinson, S. (1998). New inequalities? Educational markets and ethnic minorities. Race, Ethnicity and Education, 1(2), 207-223. https://doi.org/10.1080/1361332980010205

UNESCO. (2019). Migración, desplazamiento y educación: Construyendo puentes, no muros. Recuperado de https://es.unesco.org/gem-report/node/1878

UNICEF. (2011). La voz de los niños, niñas y adolescentes y discriminación. Recuperado de http://www.unicef.cl/pdf/PPTLaVozDiscriminacion2011.pdf

Valenzuela, J. P., Bellei, C. y De los Ríos, D. (2014). Socioeconomic school segregation in a market oriented educational system. The case of Chile. Journal of Education Policy, 29(2), 217-241. https://doi.org/10.1080/02680939.2013.806995 
Van Zanten, A. (1997). Schooling inmigrants in France in the 1990s: Success or failure of the republican model of integration? Anthropology \& Education Quarterly, 28(3), 35 1-374.

https://doi.org/10.1525/aeq.1997.28.3.351

Van Zanten, A. (2003). Middle class parents and social mix in French urban schools: Reproduction and transformation of class relations in education. International Studies in Sociology of Education, 3(2), 107-123. https://doi.org/10.1080/09620210300200106

Van Zanten, A. (2015). The determinants and dynamics of school choice. A comparative review. En P. Seppänen, A, Carrasco, M., Kalalahti, R. Rinne y H. Simola (Eds.), Contrasting dynamics in education politics of extremes. School choice in Chile and Finland (pp. 3-28). Rotterdam: Sense Publishers.

Verger, A., Bonal, X. y Zancajo, A. (2016). Recontextualización de políticas y (cuasi) mercados educativos. Un análisis de las dinámicas de demanda y oferta escolar en Chile. Education Policy Analysis Archives, 24(27), 1-27. https://doi.org/10.14507/epaa.24.2098

\section{Anexos}

Anexo 1. Resultados escuelas A y B en SIMCE 2006-2017, $4^{\circ}$ básico

\begin{tabular}{ccccc}
\hline \multirow{2}{*}{ AÑo } & \multicolumn{2}{c}{ COMPRENSIÓn LECTORA } & \multicolumn{2}{c}{ MATEMÁTICAS } \\
\cline { 2 - 5 } & Escuela A & Escuela B & Escuela A & Escuela B \\
\hline 2006 & 217 & 263 & 219 & 252 \\
2008 & 237 & 270 & 247 & 242 \\
2009 & 213 & 268 & 221 & 260 \\
2010 & 226 & 280 & 220 & 254 \\
2011 & 226 & 277 & 220 & 273 \\
2012 & 215 & 276 & 219 & 272 \\
2013 & 230 & 267 & 222 & 258 \\
2014 & 212 & 263 & 229 & 254 \\
2015 & 263 & 255 & 262 & 247 \\
2016 & 265 & 254 & 254 & 250 \\
2017 & 264 & 257 & 254 & 244 \\
\hline
\end{tabular}

Fuente: Elaboración propia en base a Información Estadística de Establecimientos, Datos Abiertos Centro de Estudios, Ministerio de Educación.

Anexo 2. Evolución de matrícula en enseñanza básica, 2004-2017

\begin{tabular}{ccc}
\hline AÑO & ESCUELA A & ESCUELA B \\
\hline 2004 & 540 & 1326 \\
2005 & 547 & 1316 \\
2006 & 504 & 1191 \\
2007 & 415 & 1138 \\
2008 & 469 & 1036 \\
2009 & 404 & 998 \\
2010 & 364 & 900 \\
2011 & 336 & 853 \\
2012 & 318 & 809 \\
2013 & 276 & 747 \\
2014 & 276 & 667 \\
2015 & 257 & 611 \\
2016 & 250 & 580 \\
2017 & 263 & 634 \\
\hline
\end{tabular}

Fuente: Elaboración propia en base a Información Estadística de Establecimientos, Datos Abiertos Centro de Estudios, Ministerio de Educación. 
Anexo 3. Mediana distancia casa - escuela y Valor p, Escuelas UGA 1

\begin{tabular}{lcc}
\hline & MEdiANA (METROS) & VALOR P \\
KRUSKAL WALIS
\end{tabular}

\section{Breve CV de las autoras}

\section{Claudia Córdoba}

Psicóloga de la Universidad de Chile y Doctora en Sociología de la Educación por la Universidad Complutense de Madrid. Académica del Departamento de Educación de la Facultad de Humanidades de la Universidad de Santiago de Chile. Sus intereses de investigación han estado alrededor de elección de escuela y segregación socio económica entre establecimientos educativos, habiendo participado también en estudios sobre iniciación profesional docente. Actualmente desarrolla proyectos de investigación centrados en la elección de escuela por parte de familias migrantes y concentración de este alumnado en escuelas de la Región Metropolitana. ORCID ID: https://orcid.org/oooo0003-0793-3420. Email: claudia.cordoba.c@usach.cl

\section{Carolina Altamirano}

Antropóloga Social de la Universidad Academia de Humanismo Cristiano, Chile. Magister en Geografía, mención intervención ambiental y territorial de la misma universidad. Se desempeña como profesional en la Comuna de Estación Central (Región Metropolitana), coordinando programas vinculados a educación, migración e infancia. Ha participado en diferentes estudios relativos a migración destacándose entre ellos "Migración China en Chile: relaciones conflicto y territorio" y "Espacialidades transfronterizas en el desierto de Atacama, movilidad y reconfiguración de identidades nacionales y étnicas". Actualmente es Tesista en el proyecto FONDECYT 1170236 "Turismo y pueblos indígenas. Estudio de discursos, prácticas y políticas públicas en tres territorios de Chile”. ORCID ID: https://orcid.org/0000-0003-1886-458X. Email: carotrini.altamirano@gmail.com

\section{Karina Rojas}

Graduado en Matemáticas con mención en Estadística de la Pontificia Universidad Católica de Chile y Doctorado en Métodos Matemático Estadísticos y Computacionales para el tratamiento de la información por la Universidad Complutense de Madrid. Diez años de experiencia en diseño y ejecución de métodos de investigación social y de mercado, tanto en entidades privadas como públicas, además de utilización de técnicas de data mining para la selección y entrega de becas sociales. Cinco años de experiencia académica impartiendo estadística, métodos estadísticos para la gestión, data mining e investigación en universidades chilenas y españolas. Participación activa en equipos de investigación en distintas universidades chilenas y españolas, tanto en el área de educación, psicología y políticas públicas, como en matemáticas, teoría de la decisión y lógica borrosa. ORCID ID: https://orcid.org/0000-0001-8747-0504.Email: karinah.rpatuelli@urjc.es 